\title{
A new late-surviving early diverging Ibero-Armorican duck-billed dinosaur and the role of the Late Cretaceous European Archipelago in hadrosauroid biogeography
}

\author{
Albert Prieto-Márquez and Miguel Ángel Carrera Farias \\ Acta Palaeontologica Polonica 66 (2), 2021: 425-435 doi:https://doi.org/10.4202/app.00821.2020
}

The anatomy and phylogenetic systematics of a well preserved hadrosauroid dentary collected in the 1990s from uppermost Maastrichtian strata (within chron C29r) of the Figuerola Formation, in the Àger syncline of the southern Pyrenees in northeastern Spain are revised. The specimen represents a new genus and species of basally branching hadrosauroid dinosaur, Fylax thyrakolasus gen. et sp. nov. This taxon is the third hadrosaurid outgroup species erected in Europe so far and the stratigraphically youngest non-hadrosaurid hadrosauroid known to date. It is diagnosed on the basis of a unique combination of characters such as a dorsal region of the coronoid process that is at least as wide anteroposteriorly as $30 \%$ of dental battery length, a coronoid process inclined anteriorly less than $80^{\circ}$ relative to the alveolar margin of the dentary, steeply inclined and flat occlusal surface of the dental battery, and dentary tooth crowns 2.8-3.3 times taller than wide without marginal denticles and with a pair of major long ridges on the enameled lingual surface. The parsimony analysis confirmed that $F$. thyrakolasus gen. et sp. nov. is a close outgroup to Hadrosauridae. However, we also found that it is sister to the hadrosauroid Tethyshadros insularis form the late Campanian-early Maastrichtian Adriatic-Dinaric Carbonate Platform (present-day Italy). Maximum likelihood reconstruction of ancestral areas on the time calibrated phylogeny revealed that the ancestry of the Fylax-Tethyshadros clade has Appalachian roots. This study supports an Asian origin for hadrosauroids and subsequent dispersal to Appalachia, where Hadrosauridae likely originated. In this scenario, the Late Cretaceous European Archipelago could have facilitated the dispersal of hadrosaurid outgroups from Asia to Appalachia.

Key words: Dinosauria, Hadrosauroidea, anatomy, systematic, end-Cretaceous, Spain, Pyrenees.

Albert Prieto-Márquez [albert.prieto@icp.cat], Institut Català de

Paleontologia Miquel Crusafont, Universitat Autònoma de

Barcelona, c/Escola Industrial 23, 08201 Sabadell, Barcelona,

Spain. Miguel Angel Carrera Farias [miguelangel.carrera@e-campus.uab.cat], Universitat Autònoma de Barcelona, Facultat de Ciències i Biociències, Entrada Sud Edifici de Ciències, Carrer de la Vall Moronta, 08193 Bellaterra (Cerdanyola del Vallès), Barcelona. 
This is an open-access article distributed under the terms of the Creative Commons

Attribution License (for details please see creativecommons.org), which permits unrestricted use, distribution, and reproduction in any medium, provided the original author and source are credited.

FoFif Full text $(1,046.6 \mathrm{kB})$ ।

Far Supplementary file $(188.8 \mathrm{kB})$ 